\title{
A NOTE ON NEW GENERATING RELATIONS FOR FUNCTIONS OF SEVERAL VARIABLES
}

\author{
D. P. SHUKLA ${ }^{1}$
}

\begin{abstract}
In this paper we have derived generating relations for functions of several variables by using the operator $T_{k}=x(k+x D)$ and the operational relations involving this operator. Some recent results of Srivastava and Panda [7] have been conveniently obtained by this method as well as some hitherto unknown results established.
\end{abstract}

Introduction. Lauricella's function of several variables is defined as follows

$$
\begin{aligned}
& F_{D}^{(r)}\left[\left(\alpha ; m_{i}\right):\left(\beta_{i} ; \phi_{i}\right) ;\left(\gamma ; \Psi_{i}\right) ; x_{1}, \ldots, x_{r}\right] \\
& \quad=\sum_{k_{1}, \ldots, k_{r}=0}^{\infty} \frac{(\alpha)_{\varepsilon m_{i} k_{i}}\left(\beta_{1}\right) k_{1} \phi_{1} \cdots\left(\beta_{r}\right)_{k_{r} \phi_{r}}}{(\gamma)_{\varepsilon k_{i} \Psi_{i}}} \cdot \frac{x_{1}^{k_{1}} \cdots x_{r}^{k_{r}}}{k_{1} ! \cdots k_{r} !},
\end{aligned}
$$

where the parameters $\alpha, \beta, \gamma$ are arbitrary complex numbers, the coefficients $m_{i}, \phi_{i}, \Psi_{i}$ are real and positive and for the convergence of multiple series

$$
1+\Psi_{i}-m_{i}-\phi_{i} \geqslant 0, \quad i=1,2, \ldots, r,
$$

the equality holds only if $\left|x_{1}\right|,\left|x_{2}\right|, \ldots,\left|x_{r}\right|$ are constrained appropriately. In particular if, $m_{i}=m, \Psi_{i}=\Psi, i=1,2, \ldots, r$, then the equality in (1.2) will hold when either

$$
m>\Psi \text { and } \sum_{i=1}^{r}\left(\frac{\left|x_{i}\right|}{G_{i}}\right)^{1 /(m-\Psi)}<1
$$

or

$$
m \leqslant \Psi \text { and } \max _{1 \leqslant i \leqslant r}\left\{\frac{\left|x_{i}\right|}{G_{i}}\right\}<1
$$

where

$$
G_{i}=m^{-m} \phi_{i}^{-\phi_{i}} \Psi^{\Psi}, \quad i=1,2, \ldots, r
$$

Received by the editors July 20, 1982.

1980 Mathematics Subject Classification. Primary 33A65.

Key words and phrases. Lauricella's functions of several variables, generating relation.

${ }^{1}$ Research supported by Council of Scientific and Industrial Research Government of India, Grant No. 13 (2707-A)/Pool. 
In this paper we have found new generating relations for Lauricella's function and some related function of several variables, viz.

$$
\begin{aligned}
\sum_{n_{1}, \ldots, n_{r}=0}^{\infty} \frac{\left(\lambda_{1}\right)_{2 n_{1}} \cdots\left(\lambda_{r}\right)_{2 n_{r}} t_{1}^{n_{1}} \cdots t_{r}^{n_{r}}}{\left(\lambda_{1}\right)_{n_{1}} \cdots\left(\lambda_{r}\right)_{n_{r}} n_{1} ! \cdots n_{r} !} & \cdot F_{D}^{(r)}\left[\left(\lambda ; m_{i}\right):\left(-n ; m_{i}\right) ;\left(\gamma ; \Psi_{i}\right) ; x_{1}, \ldots, x_{r}\right] \\
= & \prod_{i=1}^{r}\left\{\left(1-4 t_{i}\right)^{-1 / 2}\left[\frac{2}{1+\left(1-4 t_{i}\right)^{1 / 2}}\right]^{\lambda_{i}-1}\right\} \\
\cdot F_{D}^{(r)}\left[\left(\lambda ; m_{i}\right):\left(1-\lambda_{i}-2 n_{i} ; m_{i}\right) ;\left(\gamma ; \Psi_{i}\right) ;\right. & \left.\left.\left.x_{1}^{m_{1}}, \ldots, x_{r}\left(\frac{2 t_{1}}{1+\left(1-4 t_{1}\right)^{1 / 2}}\right)^{m_{r}}, 4 t_{r}\right)^{1 / 2}\right)^{m_{r}}\right] .
\end{aligned}
$$

Recently Srivastava and Panda [7] considered the functions

$$
f\left[z_{1}, \ldots, z_{r}\right]=\sum_{k_{1}, \ldots, k_{r}=0}^{\infty} C\left(k_{1}, \ldots, k_{r}\right) \frac{z_{1}^{k_{1}}}{k_{1} !} \cdots \frac{z_{r}^{k_{r}}}{k_{r} !},
$$

where the coefficients $C\left(k_{1}, \ldots, k_{r}\right), k_{i} \geqslant 0, i=1,2, \ldots, r$, are arbitrary constants real or complex.

$$
\begin{aligned}
\Delta_{n_{1}, \ldots, n_{r}: q_{1}, \ldots, q_{r}}^{\left(\alpha_{1}, \ldots, \boldsymbol{\alpha}_{r}: \boldsymbol{\beta}_{1}, \ldots, \boldsymbol{\beta}_{r}\right)}\left(z_{1}, \ldots, z_{r}\right)= & \sum_{k_{1}=0}^{\left[n_{1} / q_{1}\right]} \ldots \sum_{k_{r}=0}^{\left[n_{r} / q_{r}\right]} C\left(k_{1}, \ldots, k_{r}\right) \\
& \cdot \prod_{i=1}^{r}\left\{\frac{\left(-n_{i}\right)_{q_{i} k_{1}}}{\left(1+\alpha_{i}+\beta_{i} n_{i}\right)_{q_{i} k_{l}}} \frac{z_{i}^{k_{1}}}{k_{i} !}\right\}
\end{aligned}
$$

where $\alpha_{i}$ and $\beta_{i}$ are parameters independent of $n_{1}, \ldots, n_{r}$ and $q_{i}$ are arbitrary positive integers, $i=1, \ldots, r$ and

$$
\begin{array}{r}
S_{n_{1}, \ldots, n_{r}: q_{1}, \ldots, q_{r}}^{\left(\alpha_{1} \ldots, \beta_{r}\right)}\left[\lambda_{1}, \ldots, \lambda_{r}: z_{1}, \ldots, z_{r}\right]=\sum_{k_{1}=0}^{\left[n_{1} / q_{1}\right]} \ldots \sum_{k_{r}=0}^{\left[n_{r} / q_{r}\right]} C\left(k_{1}, \ldots, k_{r}\right) \\
\cdot \prod_{i=1}^{r}\left\{\frac{\left(-n_{i}\right)_{q_{i} k_{i}}\left(1+\alpha_{i}+\left(\beta_{i}+1\right) n_{i}\right)_{\lambda_{i} k_{i}}}{\left(1+\alpha_{i}+\beta_{i} n_{i}\right)_{\left(\lambda_{i}+q_{i}\right) k_{i}}} \frac{z_{i}^{k_{i}}}{k_{i} !}\right\}
\end{array}
$$

where $\alpha_{i}, \beta_{i}$ and $\lambda_{i}, i=1,2, \ldots, r$, are complex parameters independent of $n_{1}, \ldots, n_{r}$ and $q_{1}, \ldots, q_{r}$ are arbitrary positive integers and derived the generating relation for them.

Besides these Srivastava [5, 6], Sharma and Abiodun [1] and Carlitz and Srivastava [10] derived generating relations for (1.7) and the $G$ function and obtained generating functions for Lauricella's function of several variables. Shukla [2] using the operator $T_{k}=x(k+x D)$ and the operational relations involving these operators derived the results of Srivastava [6], Sharma and Abiodun [1] and Carlitz and Srivastava [10] and established some hitherto unknown results. 
In this paper we propose to derive the results of Srivastava and Panda [7] and other results by making use of operator formulas (2.1), (3.1), (3.4), (3.7) and (3.9).

In $\$ 2$ we obtain generating relations for functions defined by (1.8) and (1.9) and in $\$ 3$ we derive various generating functions for Lauricella's function of several variables.

2. Generating relations for functions of several variables. In this section we shall use the Mittal [4] operational generating formula

$$
\sum_{n=0}^{\infty} \frac{1}{n !} T_{a+1+(m-1) n}^{n}\{f(x)\}=\frac{(1+\nu)^{a+1}}{1-(m-1) \nu} f[x(1+\nu)],
$$

where $\nu=x(1+\nu)^{m}, m$ being constant and $f(x)$ admits a formal power series in $x$ and $T_{k} \equiv x(k+x D), D=d / d x$.

Assuming that the operator $T_{1, k}$ is the operator $T_{k}$ operating on $t_{1}$ alone and similarly $T_{r, k}$ the operator on $t_{r}$ alone and replacing $a$ by $a_{1}=\alpha_{1}, \ldots, a_{r}=\alpha_{r}, m$ by $m_{1}=\beta_{1}+1, \ldots, m_{r}=\beta_{r}+1, n$ by $n_{1}, n_{2}, \ldots, n_{r}$ respectively and putting

$$
f(x) \equiv f\left[x_{1}\left(-y_{1}^{-\beta_{1}} t_{1}^{\beta_{1}+1}\right)^{m_{1}}, \ldots, x_{r}\left(-y_{r}^{-\beta_{r}} t_{r}^{\beta_{r}+1}\right)^{m_{r}}\right]
$$

in (2.1) $r$ times, we easily get

$$
\begin{aligned}
& \sum_{n_{1} \ldots, n_{r}=0}^{\infty}\left(\begin{array}{c}
\alpha_{1}+\left(\beta_{1}+1\right) n_{1} \\
n_{1}
\end{array}\right) \cdots\left(\begin{array}{c}
\alpha_{r}+\left(\beta_{r}+1\right) n_{r} \\
n_{r}
\end{array}\right) t_{1}^{n_{1}} t_{2}^{n_{2}} \cdots t_{r}^{n_{r}} \\
& \cdot \sum_{k_{1}, \ldots, k_{r}=0}^{N} C\left(k_{1}, \ldots, k_{r}\right) \prod_{i=1}^{r}\left\{\frac{\left(-n_{i}\right)_{m_{i} k_{i}}}{\left(1+\alpha_{i}+\beta_{i} n_{i}\right)_{m_{i} k_{i}}} \frac{x_{i}^{k_{i}} y_{i}^{-\beta_{i} m_{i}} t_{i}^{\left(1+\beta_{i}\right) m_{i}}}{k_{i} !}\right\} \\
& =\frac{\left(1+\nu_{1}\right)^{\alpha_{1}+1} \cdots\left(1+\nu_{r}\right)^{\alpha_{r}+1}}{\left(1-\beta_{1} \nu_{1}\right) \cdots\left(1-\beta_{1} \nu_{r}\right)} f\left[x_{1}\left[-y_{1}^{-m_{1} \beta_{1}}\left\{t_{1}^{\beta_{1}+1}\left(1+\nu_{1}\right)^{\beta_{i}+1}\right\}^{m_{1}}\right], \ldots\right. \text {, } \\
& \left.x_{r}\left[-y^{-m_{r} \beta_{r}}\left\{t_{r}^{\beta_{r}+1}\left(1+\nu_{r}\right)^{\beta_{r}+1}\right\}^{m_{r}}\right]\right],
\end{aligned}
$$

where $\nu_{i}=t_{i}\left(1+\nu_{i}\right)^{\beta_{i}+1}, i=1,2, \ldots, r$, and $N=\min \left(n_{1}, n_{2}, \ldots, n_{r}\right)$. Now putting $y_{i}=t_{i}, i=1,2, \ldots, r$, in (2.2) we get

$$
\begin{aligned}
\sum_{n_{1}, \ldots, n_{r}=0}^{\infty}\left(\begin{array}{c}
\alpha_{1}+ \\
n_{1}
\end{array}\right. & \left.\left(\beta_{1}+1\right) n_{1}\right) \ldots\left(\begin{array}{c}
\alpha_{r}+\left(\beta_{r}+1\right) n_{r} \\
n_{r}
\end{array}\right) \\
& \cdot \Delta_{\left.n_{1}, \ldots, n_{r}: \boldsymbol{\alpha}_{1}: \ldots, \boldsymbol{\beta}_{1}, \ldots, \boldsymbol{\beta}_{r}\right)}^{\left(\alpha_{1}\right)}\left(x_{1}, \ldots, x_{r}\right) t_{1}^{n_{1}} \cdots t_{r}^{n_{r}} \\
= & \frac{\left(1+\nu_{1}\right)^{\alpha_{1}+1} \cdots\left(1+\nu_{r}\right)^{\alpha_{r}+1}}{\left(1-\beta_{1} \nu_{1}\right) \cdots\left(1-\beta_{r} \nu_{r}\right)} f\left[x_{1}\left(-\nu_{1}\right)^{m_{1}}, \ldots, x_{r}\left(-\nu_{r}\right)^{m_{r}}\right],
\end{aligned}
$$

where the function $\Delta$ is given by (1.8), which is due to Srivastava and Panda [7, Theorem 2]. Further with the similar assumptions on operator $T_{k}, a$ and $m$ as in the derivation of (2.3), but taking

$$
f(x) \equiv f\left[x_{1}(-1)^{m_{1}} y_{1}^{-\beta_{1} m_{1}-\lambda_{1}} t_{1}^{\left(1+\beta_{1}\right) m_{1}+\lambda_{1}}, \ldots, x_{r}(-1)^{m_{r}} y_{r}^{-\beta_{r} m_{r}-\lambda_{r}} \cdot t_{r}^{\left(1+\beta_{r}\right) m_{r}+\lambda_{r}}\right]
$$


in (2.1) and using it $r$ times, we similarly have

$$
\begin{aligned}
& \sum_{n_{1}, \ldots, n_{r}=0}^{\infty}\left(\begin{array}{c}
\alpha_{1}+\left(\beta_{1}+1\right) n_{1} \\
n_{1}
\end{array}\right) \ldots\left(\begin{array}{c}
\alpha_{r}+\left(\beta_{r}+1\right) n_{r} \\
n_{r}
\end{array}\right) \\
& \cdot S_{\left.n_{1}, \ldots, n_{r}: \boldsymbol{\alpha}_{1}, \boldsymbol{\beta}_{1}, \ldots, m_{r}\right)}^{\left(\alpha_{1}, \beta_{r}\right)}\left[\lambda_{1}, \ldots, \lambda_{r}: x_{1}, \ldots, x_{r}\right] t_{1}^{n_{1}} \ldots t_{r}^{n_{r}} \\
= & \prod_{i=1}^{r}\left\{\frac{\left(1+\nu_{i}\right)^{\alpha_{i}+1}}{\left(1-\beta_{1} \nu_{i}\right)}\right\} f\left[x_{1}\left(-\nu_{1}\right)^{m_{1}}\left(1+\nu_{1}\right)^{\lambda_{1}}, \ldots, x_{r}\left(-\nu_{r}\right)^{m_{r}}\left(1+\nu_{r}\right)^{\lambda_{r}}\right],
\end{aligned}
$$

where $\nu_{i}=t_{i}\left(1+\nu_{i}\right)^{\beta_{i}+1}, i=1,2, \ldots, r$, and the function $S$ is given by (1.9). This is also due to Srivastava and Panda [7, Theorem 2.1].

3. Lauricella's function of several variables. We now make use of the Mittal [3] operational formula

$$
\sum_{n=0}^{\infty} \frac{1}{n !} T_{a+1}^{n}\{f(x)\}=(1-x)^{-a-1} f\left[\frac{x}{1-x}\right],
$$

where $f(x)$ admits a formal power series in $x$. Replacing $a$ by $a_{1}=\lambda_{1}-1, \ldots, a_{r}=$ $\lambda_{r}-1, n$ by $n_{1}, \ldots, n_{r}$ and assuming that $T_{i, k}$ is the operator $T_{k}$ operating on $t_{i}$ alone for $i=1,2, \ldots, r$ respectively and putting

$$
f(x) \equiv F_{D}^{(r)}\left[\left(\lambda ; m_{i}\right):\left(\beta_{i} ; \phi_{i}\right) ;\left(\gamma ; \Psi_{i}\right) ;\left(-t_{1}\right)^{m_{1}} x_{1}, \ldots, x_{r}\left(-t_{r}\right)^{m_{r}}\right]
$$

in (3.1) and using it $r$ times, we get

$$
\begin{aligned}
\sum_{n_{1}, \ldots, n_{r}=0}^{\infty} & \frac{\left(\lambda_{1}\right)_{n_{1}} \cdots\left(\lambda_{r}\right)_{n_{r}} t_{1}^{n_{1}} \cdots t_{r}^{n_{r}}}{n_{1} ! \cdots n_{r} !} \\
& \sum_{k_{1}, \ldots, k_{r}=0}^{N} \frac{(\lambda)_{\varepsilon m_{i} k_{i}}\left(-n_{1}\right)_{m_{1} k_{1}} \cdots\left(-n_{r}\right)_{m_{r} k_{r}}\left(\beta_{1}\right)_{k_{1} \phi_{1}} \cdots\left(\beta_{r}\right)_{k_{r} \phi_{r}}}{(\gamma)_{\varepsilon \Psi_{i} k_{i}}\left(\lambda_{1}\right)_{m_{1} k_{1}} \cdots\left(\lambda_{r}\right)_{m_{r} k_{r}}} \frac{x_{1}^{k_{1}} \cdots x_{r}^{k_{r}}}{k_{1} ! \cdots k_{r} !} \\
= & \prod_{i=1}^{r}\left\{\left(1-t_{i}\right)^{\lambda_{i}}\right\} F_{D}^{(r)}\left[\left(\lambda ; m_{i}\right):\left(\beta_{i} ; \phi_{i}\right) ;\left(\gamma ; \Psi_{i}\right) ; \quad x_{1}\left(\frac{t_{1}}{t_{1}-1}\right)^{m_{1}}, \ldots, x_{r}\left(\frac{t_{r}}{t_{r}-1}\right)^{m_{r}}\right] .
\end{aligned}
$$

Taking $\beta_{r}=\lambda_{r}$ and $\phi_{r}=m_{r}$ in (3.2) we have the generating relation

$$
\begin{aligned}
\sum_{n_{1}, \ldots, n_{r}=0}^{\infty} & \frac{\left(\lambda_{1}\right)_{n_{1}} \cdots\left(\lambda_{r}\right)_{n_{r}} t_{1}^{n_{1}} \cdots t_{r}^{n_{r}}}{n_{1} ! \cdots n_{r} !} F_{D}^{(r)}\left[\left(\lambda ; m_{1}\right):\left(-n_{i} ; m_{1}\right) ;\left(\gamma ; \Psi_{i}\right) ; x_{1}, \ldots, x_{r}\right] \\
= & \prod_{i=1}^{r}\left\{\left(1-t_{i}\right)^{\lambda_{i}}\right\} F_{D}^{(r)}\left[\left(\lambda ; m_{i}\right) ;\left(\lambda_{i}, m_{i}\right) ;\left(\gamma ; \Psi_{i}\right) ;\right. \\
& \left.x_{1}\left(\frac{t_{1}}{t_{1}-1}\right)^{m_{1}}, \ldots, x_{r}\left(\frac{t_{r}}{t_{r}-1}\right)^{m_{r}}\right] .
\end{aligned}
$$


Again by making use of the Mittal [3] operational formula

$$
\begin{aligned}
\sum_{n=0}^{\infty} \frac{1}{n !} T_{a+n}^{n}\{f(x)\}= & (1-4 x)^{-1 / 2}\left[\frac{2}{1+(1-4 x)^{1 / 2}}\right]^{a-1} \\
& \cdot f\left[\frac{2 x}{1+(1-4 x)^{1 / 2}}\right]
\end{aligned}
$$

replacing $a$ by $a_{1}=\lambda_{1}, \ldots, a_{r}=\lambda_{r}, n$ by $n_{1}, \ldots, n_{r}$ and assuming that $T_{i, k}$ is the operator $T_{k}$ operating on $t_{i}$ alone, $i=1,2, \ldots, r$, respectively and taking

$$
f(x) \equiv F_{D}^{(r)}\left[\left(\lambda ; m_{i}\right) ;\left(\beta_{i} ; \phi_{i}\right) ;\left(\gamma ; \Psi_{i}\right) ; x_{1} t_{1}^{m_{1}}, \ldots, x_{r} t_{r}^{m_{r}}\right]
$$

in (3.4) and using it $r$ times, we have the generating relation

$$
\begin{aligned}
\sum_{n_{1}, \ldots, n_{r}=0}^{\infty} & \frac{\left(\lambda_{1}\right)_{2 n_{1}} \cdots\left(\lambda_{r}\right)_{2 n_{r}} t_{1}^{n_{1}} \cdots t_{r}^{n_{r}}}{\left(\lambda_{1}\right)_{n_{1}} \cdots\left(\lambda_{r}\right)_{n_{r}} n_{1} ! \cdots n_{r} !} \\
\cdot & \sum_{k_{1}, \ldots, k_{r}=0}^{N} \frac{(\lambda)_{\varepsilon m_{i} k_{i}}\left(-n_{1}\right)_{m_{1} k_{1}} \cdots\left(-n_{r}\right)_{m_{r} k_{r}}\left(\beta_{1}\right)_{k_{1} \phi_{1}} \cdots\left(\beta_{r}\right)_{k_{r} \phi_{r}}}{(\gamma)_{\varepsilon k_{i} \Psi_{i}}\left(1-\lambda_{1}-2 n_{1}\right)_{m_{1} k_{1}} \cdots\left(1-\lambda_{r}-2 n_{r}\right)_{m_{r} k_{r}}} \frac{x_{1}^{k_{1}} \cdots x_{r}^{k_{r}}}{k_{1} ! \cdots k_{r} !} \\
= & \prod_{i=1}^{r}\left\{\left(1-4 t_{i}\right)^{-1 / 2}\left[\frac{2}{1+\left(1-4 t_{i}\right)^{1 / 2}}\right]^{\lambda_{i}-1}\right\} \\
\cdot F_{D}^{(r)}\left[\left(\lambda ; m_{i}\right):\left(\beta_{i} ; \phi_{i}\right) ;\left(\gamma ; \Psi_{i}\right) ; x_{1}\left(\frac{2 t_{1}}{1+\left(1-4 t_{1}\right)^{1 / 2}}\right)^{m_{1}}, \ldots,\right. & \left.x_{r}\left(\frac{2 t_{r}}{1+\left(1-4 t_{r}\right)^{1 / 2}}\right)^{m_{r}}\right] .
\end{aligned}
$$

Putting $\phi_{r}=m_{r}$ and $\beta_{r}=1-\lambda_{r}-2 n_{r}$ in equation (3.5), we get

$$
\begin{aligned}
\sum_{n_{1}, \ldots, n_{r}=0}^{\infty} & \frac{\left(\lambda_{1}\right)_{2 n_{1}} \cdots\left(\lambda_{r}\right)_{2 n_{r}} t_{1}^{n_{1} \cdots t_{r}} t_{r}^{n_{r}}}{\left(\lambda_{1}\right)_{n_{1}} \cdots\left(\lambda_{r}\right)_{n_{r}} n_{1} ! \cdots n_{r} !} F_{D}^{(r)}\left[\left(\lambda ; m_{i}\right):\left(-n_{i} ; m_{i}\right) ;\left(\gamma ; \Psi_{i}\right) ; x_{1}, \ldots, x_{r}\right] \\
= & \prod_{i=1}^{r}\left\{\left(1-4 t_{i}\right)^{-1 / 2}\left[\frac{2}{1+\left(1-4 t_{i}\right)^{1 / 2}}\right]^{\lambda_{i}-1}\right\} \\
& \cdot F_{D}^{(r)}\left[\left(\lambda ; m_{i}\right):\left(1-\lambda_{i}-2 n_{i} ; m_{i}\right) ;\left(\gamma ; \Psi_{i}\right) ;\right. \\
& \left.x_{1}\left(\frac{2 t_{1}}{1+\left(1-4 t_{1}\right)^{1 / 2}}\right)^{m_{1}}, \ldots, x_{r}\left(\frac{2 t_{r}}{1+\left(1-4 t_{r}\right)^{1 / 2}}\right)^{m_{r}}\right] .
\end{aligned}
$$


Again, making use of the Mittal [3] operational formula

$$
\sum_{n=0}^{\infty} \frac{1}{n !} T_{a-n}^{n}\{f(x)\}=(1+x)^{a-1} f[x(1+x)]
$$

and proceeding as above, we get the generating relation

$$
\begin{aligned}
\sum_{n_{1}, \ldots, n_{r}=0}^{\infty}(-1)^{n_{1}+\cdots+n_{r}} \prod_{i=1}^{r} \frac{\left(1-\lambda_{i}\right)_{n_{i}} t_{i}^{n_{i}}}{n_{i} !} & \\
& \cdot F_{1: 0, \ldots, 0,0}^{1: 2, \ldots}\left[\begin{array}{c}
\left.\left(\lambda ; m_{i}\right):\left(-n ; m_{i}\right),\left(\lambda_{i} ; m_{i}\right) ; x_{1}, \ldots, x_{r}\right] \\
\left(\gamma ; \Psi_{i}\right)
\end{array}\right] \\
= & \prod_{i=1}^{r}\left(1+t_{i}\right)^{\lambda_{i}-1} F_{D}^{(r)}\left[\left(\lambda ; m_{i}\right):\left(\lambda_{i}-n_{i} ; 2 m_{i}\right) ;\left(\gamma ; \Psi_{i}\right) ;\right. \\
& \left.x_{1}\left(-t_{1}\left(1+t_{1}\right)^{m_{1}}\right), \ldots, x_{r}\left(-t_{r}\left(1+t_{r}\right)^{m_{r}}\right)\right] .
\end{aligned}
$$

Next, using the Mittal [3] operational formula

$$
\sum_{n=0}^{\infty} \frac{1}{n !} T_{a-2 n}^{n}\{f(x)\}=(1+4 x)^{-1 / 2}\left[\frac{2}{1+(1+4 x)^{1 / 2}}\right]^{-a} \cdot f\left[\frac{x+(1+4 x)^{1 / 2}}{2}\right]
$$

replacing $a$ by $a_{1}=\lambda_{1}, \ldots, a_{r}=\lambda_{r}, n$ by $n_{1}, \ldots, n_{r}$ and assuming that $T_{i, k}$ is the operator $T_{k}$ operating on $t_{i}$ alone, $i=1,2, \ldots, r$, respectively and putting

$$
f(x) \equiv F_{D}^{(r)}\left[\left(\lambda ; m_{i}\right):\left(\beta_{i} ; \phi_{i}\right) ;\left(\gamma ; \Psi_{i}\right) ; x_{1}\left(\frac{27}{4} t_{1}\right)^{m_{1}}, \ldots,\left(\frac{27}{4} t_{r}\right)^{m_{r}}\right]
$$

in (3.9) and using (3.9) $r$-times we get the following generating relation:

$$
\begin{aligned}
& \sum_{n_{1}, \ldots, n_{r}=0}^{\infty} \prod_{i=1}^{r} \frac{\left(1-\lambda_{i}\right)_{2 n_{i}} t_{i}^{n_{i}}}{n_{i} !\left(1-\lambda_{i}\right)_{n_{i}}} \\
& \cdot \sum_{k_{1}, \ldots, k_{r}=0}^{N} \frac{(\lambda)_{\varepsilon m_{i} k_{i}}\left(\beta_{1}\right)_{k_{1} \phi_{1}} \cdots\left(\beta_{r}\right)_{k_{r} \phi_{r}}}{(\gamma)_{\varepsilon m_{i} k_{i}}} \cdot \frac{x_{1}^{k_{1}} \cdots x_{r}^{k_{r}}}{k_{1} ! \cdots k_{r} !} \\
& \cdot \prod_{i=1}^{r}\left[\frac{\left(\left(\lambda-n_{i}\right) / 2\right)_{m_{i} k_{i}}\left(\left(\lambda-n_{i}+1\right) / 2\right)_{m_{i} k_{i}}\left(-n_{i}\right)_{m_{i} k_{i}}}{\left(\left(\lambda-2 n_{i}\right) / 3\right)_{m_{i} k_{i}}\left(\left(\lambda-2 n_{i}+1\right) / 3\right)_{m_{i} k_{i}}\left(\left(\lambda-2 n_{i}+2\right) / 3\right)_{m_{i} k_{i}}}\right] \\
&= \prod_{i=1}^{r}\left\{\left(1+4 t_{i}\right)^{-1 / 2}\left[\frac{2}{1+\left(1+4 t_{i}\right)^{1 / 2}}\right]^{-\lambda_{i}}\right\} \\
& \cdot F_{D}^{(r)}\left[\left(\lambda ; m_{i}\right):\left(\beta_{i} ; \phi_{i}\right) ;\left(\gamma ; \Psi_{i}\right) ; x_{1}\left[\frac{27}{4} t_{1}\left\{1+\left(1+4 t_{1}\right)^{1 / 2}\right\}^{m_{1}}\right], \ldots,\right. \\
&\left.x_{r}\left[\frac{27}{4} t_{r}\left\{1+\left(1+4 t_{r}\right)^{1 / 2}\right\}^{m_{r}}\right]\right],
\end{aligned}
$$


which is a generalization of a result due to Shukla [2]. Finally replacing $a$ by $a_{1}=\lambda_{1}-1, \ldots, a_{r}=\lambda_{r}-1, n$ by $n_{1}, \ldots, n_{r}$ and assuming that $T_{i, k}$ is the operator $T_{k}$ operating on $t_{i}$ alone, $i=1,2, \ldots, r$ respectively and putting

$f(x) \equiv F_{D}^{(r)}\left[\left(\lambda ; m_{i}\right):\left(\beta_{i} ; \phi_{i}\right) ;\left(\gamma ; \Psi_{i}\right) ; x_{1}\left[\frac{(2-m)^{2-m}}{(1-m)^{1-m}} t_{1}\right]^{m_{1}}, \ldots\right.$,

$$
\left.x_{r}\left[\frac{(2-m)^{2-m}}{(1-m)^{1-m}} t_{r}\right]^{m_{r}}\right]
$$

in (2.1) and using it $r$-times we get the generating relation

$$
\begin{aligned}
& \sum_{n_{1}, \ldots, n_{r}=0}^{\infty}(-1)^{n_{1}+\cdots+n_{r}} \prod_{i=1}^{r} \frac{\left(\lambda_{i}\right)_{m n_{i}} t_{i}^{n_{i}}}{n_{i} !\left(\lambda_{i}\right)_{(m-1) n_{i}}} \\
& \cdot \sum_{k_{1}, \ldots, k_{r}=0}^{N} \frac{(\lambda)_{\varepsilon m_{i} k_{i}}\left(\beta_{1}\right)_{k_{1} \phi_{1}} \cdots\left(\beta_{r}\right)_{k_{r} \phi_{r}} x_{1}^{k_{1}} \cdots x_{r}^{k_{r}}}{(\gamma)_{\varepsilon k_{i} \Psi_{i} k_{1} ! \cdots k_{r} !}} \\
& \cdot \prod_{i=1}^{r}\left[\frac{\left(-n_{i}\right)_{m_{i} k_{i}}\left(\left(\lambda+m n_{i}\right) /(1-m)\right)_{m_{i} k_{i}} \cdots}{\left(\left(\lambda+(m-1) n_{i}\right) /(2-m)\right)_{m_{i} k_{i}} \cdots}\right. \\
&=\left.\frac{\left(\left(\lambda+m n_{i}-m\right) /(1-m)\right)_{m_{i} k_{i}}}{\left(\left(\lambda+(m-1) n_{i}+1-m\right) /(2-m)\right)_{m_{i} k_{i}}}\right] \\
& \prod_{i=1}^{r} \frac{\left(1+\nu_{i}\right)^{\lambda_{i}}}{\left(1-(m-1) \nu_{i}\right)} F_{D}^{(r)}\left[\left(\lambda ; m_{i}\right):\left(\beta_{i} ; \phi_{i}\right) ;\left(\gamma ; \Psi_{i}\right) ;\right. \\
& x_{1}\left[\frac{(2-m)^{2-m}}{\left.(1-m)^{1-m} t_{1}\left(1+\nu_{1}\right)\right]^{m_{1}}, \ldots,}, \ldots\right] \\
&\left.x_{r}\left[\frac{(2-m)^{2-m}}{(1-m)^{1-m}} t_{r}\left(1+\nu_{r}\right)\right]^{m_{r}}\right]
\end{aligned}
$$

where $\nu_{i}=t_{i}\left(1+\nu_{i}\right)^{m}, i=1,2, \ldots, r$, which is again a generalization of a result due to Shukla [2].

\section{REFERENCES}

1. B. L. Sharma and R. F. Abiodun, Generating functions for generalized function of two variables, Proc. Amer. Math. Soc. 46 (1974), 69-72.

2. D. P. Shukla, A note on generating relations for Lauricella's function of several variables, Proc. Amer. Math. Soc. 73 (1979), 199-206.

3. H. B. Mittal, Some generating functions, Univ. Lisboa Revista Fac. Ci. A (2) 13 (1969/70), 43-54.

4. Polynomials defined by generating relations, Trans. Amer. Math. Soc. 168 (1972), 73-84.

5. H. M. Srivastava, A generating function for certain coefficients involving several complex variables, Proc. Nat. Acad. Sci. U.S.A. 67 (1970), 1079-1080.

6. A new class of generating functions involving several complex variables, Nederl. Akad. Wetensch. Proc. Ser. A 74 = Indag. Math. 33 (1971), 483-486. 
7. H. M. Srivastava and R. Panda, New generating functions involving several complex variables, Rend. Circ. Mat. Palermo (2) 29 (1980), 23-41.

8. H. M. Srivastava and R. G. Buschman, Some polynomials defined by generating relations, Trans. Amer. Math. Soc. 205 (1975), 360-370.

9. H. M. Srivastava and M. C. Daudst, On Eulerian integrals associated with Kampe de Feriet's function, Publ. Inst. Math. (Beograd) (N.S.) 9 (23) (1969), 199-202.

10. L. Carlitz and H. M. Srivastava, Some hypergeometric polynomials associated with Lauricella's function of several variables. I, Vestnik Leningrad Univ. Math. 13 (28) (1976), 41-47.

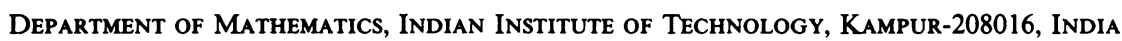

